УДК 622.411

\title{
МОДЕЛИРОВАНИЕ ДИНАМИКИ ТЕПЛОВОГО РЕЖИМА ТУПИКОВЫХ ГОРНЫХ ВЫРАБОТОК С ИСТОЧНИКОМ ВЫДЕЛЕНИЯ ТЕПЛОТЫ
}

\author{
Казаков Борис Петрович1, \\ aero_kaz@mail.ru
}

Шалимов Андрей Владимирович1, shalimovav@mail.ru

1 Горный институт УрО РАН фрилиал Пермского фредерального исследовательского центра УрО РАН, Россия, 614007, г. Пермь, ул. Сибирская, 78а.

\begin{abstract}
Актуальность исследования обусловлена необходимостью снижения температуры воздуха в тупиковых горных выработках при ведении работ, связанных с выделением большого количества теплоты. Разработка способов нормализации термодинамических параметров рудничного воздуха в забоях на основе построения прогнозных математических моделей изменения теплового режима является необходимым условием обеспечения безопасности труда в горнодобывающей промышленности. Цель: получение зависимостей для определения скорости повышения температуры воздуха в тупиковой выработке при известных величинах интенсивности выделения теплоты и скорости её отведения вентиляционной струёй.

объекты: тупиковые выработки и окружающий породный массив.

Методы: решение задачи нестационарного теплообмена рудничного воздуха и породного массива в сопряжённой постановке с помощью преобразований Лапласа; оценка безопасности по газовому фактору интенсификации выноса теплоты из горной выработки путём увеличения подачи воздуха в забой за счёт частично-повторного использования воздуха.

Результаты. Проведён анализ проблемы отвода теплоты, выделяющейся при работе оборудования в замкнутом пространстве горных выработок. Показано, что процессов поглощения теплоты породным массивом и её отведения за счёт проветривания может быть недостаточно для обеспечения нормативных значений температуры воздуха. Разработана математическая модель сопряжённого теплообмена воздуха и породного массива, позволяющая прогнозировать повышение температуры с течением времени в зависимости от длины выработки и суммарной интенсивности выделения теплоты в ней. Установлено, что теплообменные процессы продолжительностью в несколько часов могут моделироваться в приближении малых времён, что значительно упрощает расчётные зависимости. Произведён расчёт динамики теплового режима при характерном для шахтных условий наборе физических параметров задачи, результаты которого явились обоснованием сделанного приближения и подтвердили опасность быстрого увеличения температуры воздуха при недостаточной скорости выноса теплоты из выработки вентиляционной струёй. Доказано, что при необходимости дополнительного отведения теплоты можно применять источники тяги высокой производительности с частично-повторным использованием исходящего воздуха без риска увеличения содержания газов в тупиковых выработках
\end{abstract}

\section{Ключевые слова:}

Теплообмен, тупиковая выработка, породный массив, преобразования Лапласа, проветривание частично-повторное использование воздуха.

\section{Введение}

При работе механизмов часть потребляемой ими энергии переходит в теплоту, которая приводит к нагреву окружающего воздуха. На поверхности земли этот нагрев, как правило, проблем не создаёт, т. к. имеет место естественный конвективный теплообмен с атмосферой. В замкнутом же подземном пространстве возникает проблема теплоотведения, которое осуществляется движущимся по выработкам вентиляционным воздухом и теплообменом с породным массивом $[1,2]$. Способ отвода теплоты с помощью проветривания является более эффективным [3], однако в некоторых случаях интенсивность проветривания оказывается недостаточной для нормализации температурных параметров воздуха $[4,5]$. Так, при работе комбайна в забое тупиковой выработки количество выделяемой теплоты может превышать количество выносимой из забоя. В результате температура воздуха после начала работы комбайна будет постоянно повышаться, спустя некоторое время достигнет величин, недопустимых по правилам безопасности [6], и приведёт к риску возникновения аварийных ситуаций, связанных с ухудшением состояния горнорабочих $[7,8]$.

Скорость повышения температуры воздуха в пространстве тупиковой выработки зависит также и от интенсивности поглощения теплоты горными породами [9]. Оценить теплоотводящий потенциал породного массива при недостаточном проветривании тупиковой выработки можно на основе решения задачи сопряжённого теплообмена и в результате определить время непрерывной работы комбайна, в течение которого не будут нарушены нормы безопасного ведения горных работ по тепловому фактору.

Математическая модель теплообмена между воздухом и породным массивом

Тупиковая выработка моделируется цилиндрической полостью радиусом $r_{0}$, м, и длиной $L \gg r_{0}$, м. Теплообмен воздуха с массивом через боковые стенки не рассматривается, поскольку площадь их в данном приближении значительно меньше цилиндрической поверхности полости. Площадь поверхности теплообмена и объём полости составляют $S=2 \pi r_{0} L$ м $^{2}$ и 
$V=2 \pi r_{0}^{2} L \mathrm{~m}^{2}$, соответственно, источник теплоты задаётся интенсивностью тепловыделения в единицу времени на единицу объёма воздуха $W, \mathrm{BT} / \mathrm{M}^{3}$. В расчётной модели принято, что полость замкнута и выноса теплоты из неё нет, но если требуется учесть такой вынос теплоты $W^{\prime}, \mathrm{BT} / \mathrm{M}^{3}$, то источником тепла следует считать $W-W^{\prime}, \mathrm{BT} / \mathrm{M}^{3}$. Коэффициент теплоотдачи между массивом и воздухом предполагается равным бесконечности [10], также считается, что распространение теплоты внутри полости происходит мгновенно, т. е. температура воздуха в любой момент времени по всей длине одинакова. Данное приближение является корректным в случае быстрого турбулентного перемешивания воздуха, которое в шахтных условиях, безусловно, реализуется по причине интенсивного проветривания выработки и мощной тепловой адвекции, препятствующей образованию горизонтальной тепловой стратификации плотности воздуха. Это позволяет рассматривать процесс распространения теплоты только в породном массиве, а в воздушной среде учитывать лишь изменение теплосодержания в целом.

Предполагается, что температуры воздуха и массива изначально одинаковы и равны температуре породы $T_{\infty},{ }^{\circ} \mathrm{C}$ на удалении от поверхности теплового контакта, на котором температурными изменениями можно пренебречь. Требуется определить скорость увеличения температуры воздуха в полости после появления источника выделения теплоты $W$. Нестационарные задачи теплообмена такого типа, как правило, решаются методами, основанными на введении модельной характеристики, именуемой «коэффициент нестационарного теплообмена» [11]. Однако, как было установлено в [12], подобные методы имеют значительную погрешность при проведении расчётов на малых временах теплообмена порядка нескольких часов. Поскольку непрерывное время работы комбайна является величиной именно такого порядка, то используется другой, более точный, метод расчёта нестационарного теплообмена рудничного воздуха с породным массивом, предложенный в работе [12].

Уравнение теплопроводности в массиве в цилиндрических координатах имеет вид:

$$
\frac{\partial T}{\partial t}=\chi_{\mathrm{M}} \frac{1}{r} \frac{\partial}{\partial r}\left(r \frac{\partial T}{\partial r}\right),
$$

где $T=T(r, t)$ - температура массива как функция радиальной координаты $r$, м (от $r_{0}$ до $\left.\infty\right)$, и времени $t$, с; $\chi_{\mathrm{M}}-$ температуропроводность массива, $\mathrm{M}^{2} / \mathrm{c}$. Плотность теплового потока из воздуха в массив, $\mathrm{BT} / \mathrm{m}^{2}$ :

$$
j=-\left.c_{v}^{\mathrm{M}} \chi_{\mathrm{M}} \frac{\partial T}{\partial r}\right|_{r=r_{0}},
$$

где $c_{v}{ }^{\mathrm{M}}$ - объёмная теплоёмкость массива, Дж/( $\left.\mathrm{M}^{3} \cdot{ }^{\circ} \mathrm{C}\right)$. Скачок температуры на границе раздела «массиввоздух» отсутствует, поскольку коэффициент теплоотдачи предполагается равным бесконечности. Изменение теплосодержания $Q$, Дж, воздуха в полости происходит в результате уменьшения теплоты за счёт $j$ и прибыли тепла за счёт $W$ :

$$
\frac{d Q}{d t}=-j S+W \frac{V}{S} .
$$

$Q=\left.c_{v}{ }^{\mathrm{B}} T\right|_{r=r_{0} 0} V$, где $c_{v}{ }^{\mathrm{B}}-$ объёмная теплоёмкость воздуха, Дж/( $\left.\mathrm{m}^{3}{ }^{\circ} \mathrm{C}\right)$. При подстановке $Q, V, S$, выраженных через $r_{0}$, и $j$ в (1) получается:

$$
\left.\left(-\frac{W}{c_{v}^{\mathrm{B}}}+\frac{\partial T}{\partial t}\right)\right|_{r=r_{0}}=\left.\frac{2 c_{v}^{\mathrm{M}} \chi_{\mathrm{M}}}{c_{v}^{\mathrm{B}} r_{0}} \frac{\partial T}{\partial r}\right|_{r=r_{0}} .
$$

После ввода безразмерных переменных: $[r]=r_{0}$ и $[r]=r_{0}^{2} \mid \chi_{\mathrm{M}}$ уравнение (2) принимает вид:

$$
\left.\left(-a+\frac{\partial T}{\partial t}\right)\right|_{r=1}=\left.b \frac{\partial T}{\partial r}\right|_{r=1},
$$

где выделены два безразмерных комплекса:

$$
\frac{W r_{0}^{2}}{c_{v}^{\mathrm{B}} \chi_{\mathrm{M}}} \equiv a \text { и } \frac{2 c_{v}^{\mathrm{M}}}{c_{v}^{\mathrm{B}}} \equiv b .
$$

Уравнение теплопроводности в безразмерных переменных $r$ и $t$ :

$$
\frac{\partial T}{\partial t}=\frac{1}{r} \frac{\partial}{\partial r}\left(r \frac{\partial T}{\partial r}\right)
$$

В начальном условии $T$ отсчитывается от $T_{\infty}$ :

$$
T(r, t=0)=0 .
$$

Для решения уравнения теплопроводности (4) с граничным условием (3) и начальным условием (5) используется метод преобразования Лапласа, в котором производится замена $T(r, t)$ на функциюизображение $\tau(r, p)=\int_{0}^{+\infty} e^{-p t} T(r, t) d t$, что позволяет понизить размерность задачи. Сходимость интеграла обеспечивается положительным знаком комплексного параметра $p(\operatorname{Re}(p)>0)$. После проведения стандартной процедуры перехода от $T$ к $\tau$ с использованием (5) для (4) и (3) получается:

$$
\left\{\begin{array}{c}
\tau^{\prime \prime}+\frac{1}{r} \tau^{\prime}-p \tau=0 \\
\left.\left(-\frac{a}{p}+p \tau\right)\right|_{r=1}=\left.b \tau^{\prime}\right|_{r=1}
\end{array},\right.
$$

где введены обозначения

$$
\frac{d \tau}{d r} \equiv \tau^{\prime} \text { и } \frac{d^{2} \tau}{d r^{2}} \equiv \tau^{\prime \prime} .
$$

Общее решение обыкновенного дифференциального уравнения второго порядка для $\tau$ в (6) имеет вид:

$$
\tau(r, p)=c_{1} J_{0}(r \sqrt{-p})+c_{2} N_{0}(r \sqrt{-p}),
$$

где $J_{0}$ и $N_{0}$ - функции Бесселя и Неймана 0 -го порядка. Поскольку на бесконечности массив всегда остаётся «непотревоженным», то $T \rightarrow 0$ при $r \rightarrow \infty$, значит и $\tau \rightarrow 0$ при $r \rightarrow \infty$, что связывает между собой коэффициенты $c_{1}$ и $c_{2}$ :

$$
\frac{c_{2}}{c_{1}}=-\lim _{r \rightarrow \infty} \frac{J_{0}(\sqrt{-p} r)}{N_{0}(\sqrt{-p} r)} \equiv-k .
$$


Из асимптотических разложений функций $J_{0}$ и $N_{0}$ при $r \rightarrow \infty$ следует, что в (8) $k=-i$ при $\operatorname{Im}(\sqrt{-p})>0$ и $k=i$ при $\operatorname{Im}(\sqrt{-p})<0$, где $i-$ мнимая единица. Оставшийся неизвестный коэффициент находится в результате подстановки (7) в граничное условие для $\tau$ в (6). В итоге функция-изображение $\tau$ на границе раздела принимает следующий вид:

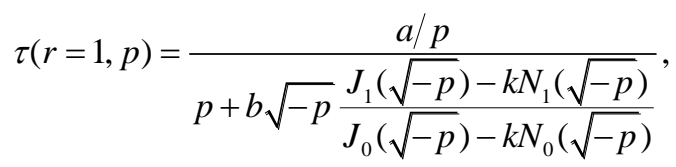

где $J_{1}$ и $N_{1}-$ функции Бесселя и Неймана 1-го порядка, полученные в результате дифференцирования $J_{0}$ и $N_{0}$. Обратное преобразование Лапласа

$$
T(r=1, t)=\frac{1}{2 \pi i} \int_{x-i \infty}^{x+i \infty} e^{p t} \tau(r=1, p) d p
$$

восстанавливает искомое значение функцииоригинала $T(r=1, t)$. Поскольку температура $T$ ограничена, то $x$ может быть любым вещественным положительным числом. Для лучшей сходимости процесса численного интегрирования оптимальной является величина $x \sim 1 / t$, при которой показатель экспоненты в (10) имеет величину порядка единицы. Реальное время непрерывной работы комбайна имеет порядок нескольких часов, и при численном расчёте оказывается, что величина $1 / t$ настолько велика, что позволяет существенно упростить выражение для функцииизображения $\tau$ с помощью асимптотических разложений функций Ганкеля $H_{n}^{(1)}(z)=J_{n}(z)+i N_{n}(z) \quad$ и $H_{n}^{(2)}(z)=J_{n}(z)-i N_{n}(z)$ при больших значениях аргумента $z$ [13]. В результате формула (9) принимает вид:

$$
\tau(r=1, p)=\frac{a / p}{p+b \sqrt{p}} .
$$

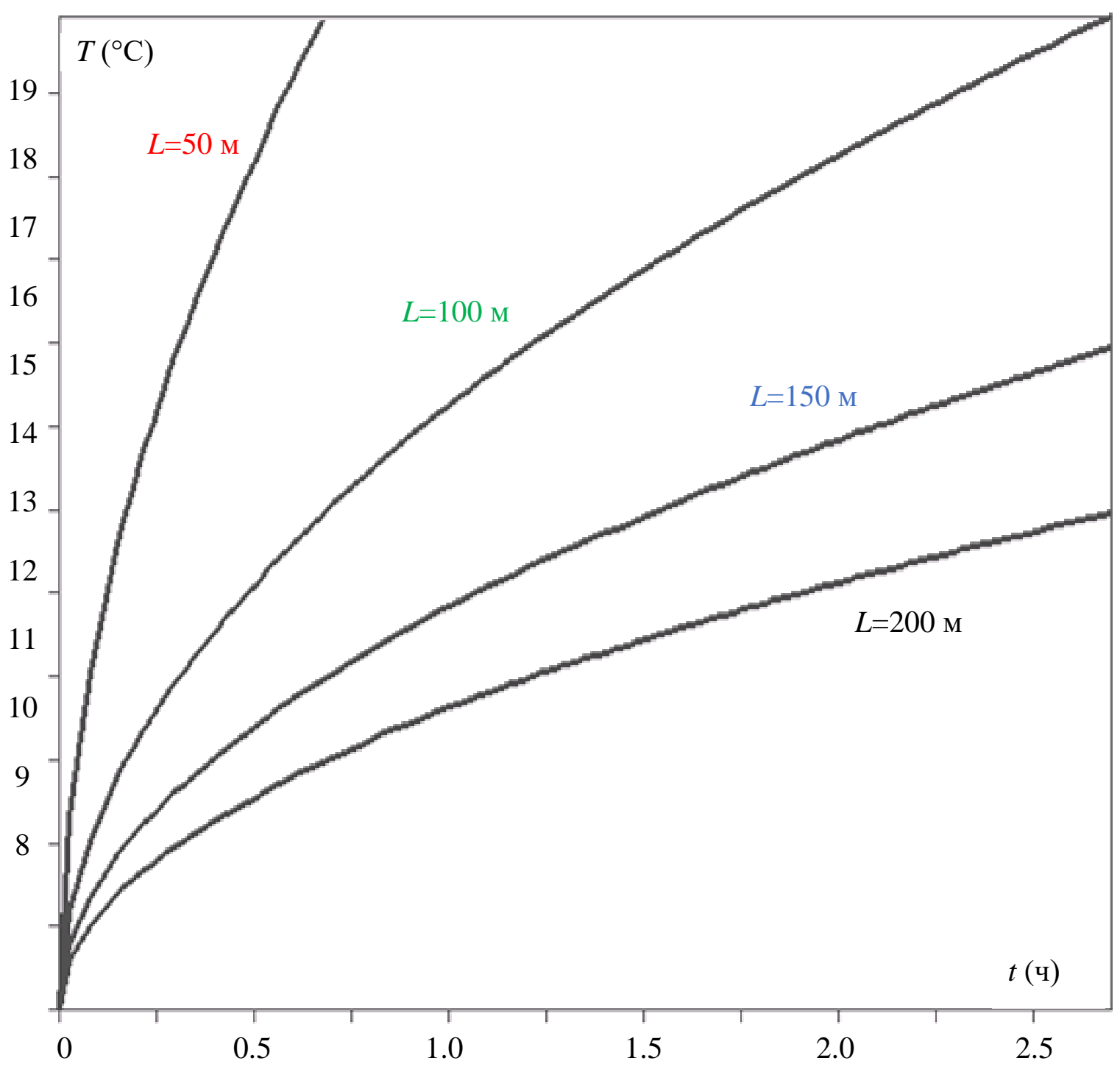

Рис. 1. Возрастание температуры воздуха со временем в зависимости от длины тупиковой выработки

Fig. 1. Increase in air temperature over time depending on the length of the dead-end workings 
Это упрощение позволяет значительно улучшить сходимость процесса численного интегрирования и увеличить скорость счёта при незначительном увеличении погрешности. Следует заметить, что данное приближение годится только для малых времён, если же рассматриваемое время теплообмена составляет несколько дней и более, то следует использовать формулу (9).

Численный расчёт температуры воздуха в зависимости от времени производился при следующем характерном наборе параметров: $c_{v}{ }^{\mathrm{M}}=1750$ кДж/( $\left.\mathrm{M}^{3}{ }^{\circ} \mathrm{C}\right)$, $c_{v}{ }^{\mathrm{B}}=1300 \mathrm{\kappa}$ Дж/( $\left.\mathrm{M}^{3} \cdot{ }^{\circ} \mathrm{C}\right), \chi_{\mathrm{m}}=5 \cdot 10^{-7} \mathrm{~m}^{2} / \mathrm{c}, r_{0}=2 \mathrm{M}, W V=500 \mathrm{\kappa BT}$. На рис. 1 представлены расчётные значения температуры при четырёх различных длинах полости $L$, м: 50, 100, 150 и 200, из которых видно, что температура воздуха в течение одного-двух часов поднимается на величину порядка $10^{\circ} \mathrm{C}$.

Интенсивность проветривания тупиковых выработок устанавливается, как правило, в соответствии с нормами безопасного содержания вредных примесей в рудничном воздухе [14]. В случае интенсивного тепловыделения, на примере приведённого выше расчёта, тепловой фактор может оказаться критичнее и потребовать большей подачи воздуха для выноса теплоты либо его предварительного охлаждения в системах кондиционирования воздуха $[15,16]$. При этом дополнительная подача воздуха с использованием части исходящей струи не должна приводить к ухудшению газовой обстановки в выработке.

\section{Частично-повторное использование воздуха для интенсификации процесса выноса теплоты из тупиковой горной выработки}

Рассматривется система, состоящая из двух горных выработок: тупиковой горной выработки и соединенной с ней сквозной горной выработки (рис. 2). В сквозной выработке задан некоторый постоянный расход воздуха $Q$. Проветривание тупиковой выработки осуществляется за счет работы вентилятора местного проветривания, установленного в сквозной выработке и имеющего вентиляционный трубопровод, протянутый до тупикового забоя.

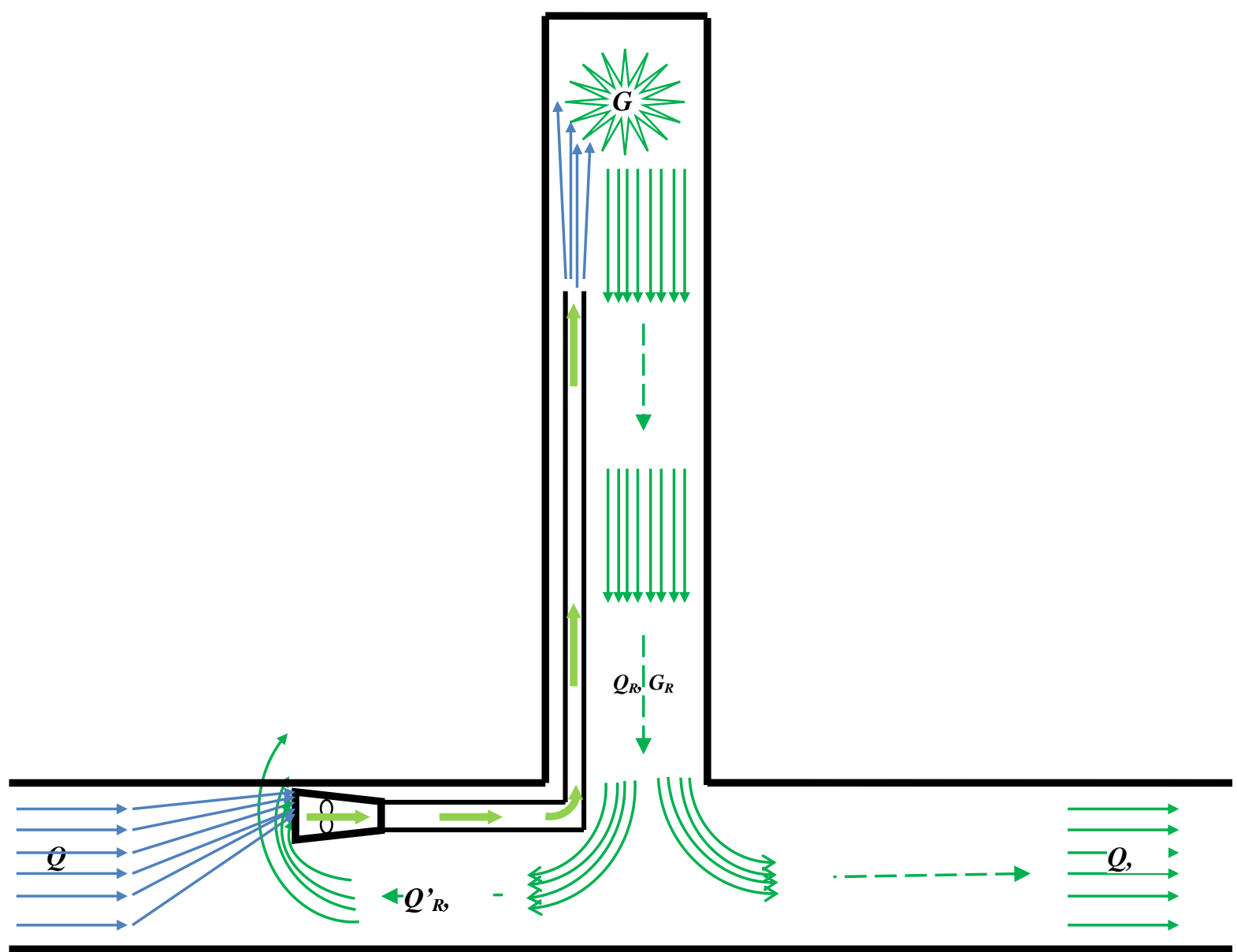

Рис. 2. Проветривание тупиковой выработки с использованием части исходящей струи

Fig. 2. Ventilation of dead-end workings using part of the outgoing jet

Согласно правилам безопасности, вентилятор местного проветривания: 1) должен быть установлен на расстоянии не менее 10 м от исходящей из устья тупиковой выработки струи отработанного (загазованного) воздуха и 2) не должен иметь производи- тельность более $70 \%$ от расхода свежего воздуха, поступающего по сквозной горной выработке. Такие ограничения введены в целях недопущения попадания исходящего воздуха обратно на всас вентилятора [17]. Действительно, при размещении вентилятора в 
устье или в самой тупиковой выработке возникнет рециркуляция с коэффициентом тем большим, чем ближе к забою расположен вентилятор. Справедливость первого ограничения не вызывает сомнений, однако целесообразность второго неочевидна. Вероятно, имеется в виду, что если производительность вентилятора приближается к общему расходу воздуха $Q$ и превышает его, то на всас вентилятора начинает поступать часть отработанного воздуха, что недопустимо по правилам безопасности [18]. Однако это не обосновано в случае, если расход свежего воздуха, подаваемого в призабойное пространство тупиковой выработки, достаточен для проветривания его по газовому фактору [19], но нет маломощного вентилятора с таким дебитом, либо требуется больше воздуха для выноса теплоты. В этом случае можно воспользоваться более мощным источником тяги, который в качестве недостающего воздуха использует часть исходящей воздушной струи. При этом концентрация вредных примесей в тупиковой выработке увеличиваться не будет, т. к. расход свежего воздуха и интенсивность газовыделений не изменяются. Этот очевидный факт можно, в качестве доказательства, наглядно продемонстрировать на основе следующей математической модели [20] рециркуляционного проветривания тупиковой выработки в нестационарной постановке (рис. 2).

В начальный момент времени $t, \mathrm{c}$, производительность вентилятора в точности равна расходу поступающего свежего воздуха в сквозной выработке $Q$, $\mathrm{m}^{3} / \mathrm{c}$. При этом выделение газа $G, \mathrm{~m}^{3} / \mathrm{c}$, в тупиковой выработке таково, что его концентрация в исходящей струе $C_{R}=G / Q$ не превышает предельно допустимой величины. В следующий момент времени производительность вентилятора увеличивается на величину расхода $Q_{R}^{\prime}, \mathrm{m}^{3} / \mathrm{c}$, который подсасывается вентилятором из исходящей струи. Требуется выяснить, как будет изменяться концентрация газа в забое. В реальных условиях объём газа в воздухе намного меньше объёма чистого воздуха, поэтому изменение объёмного расхода воздуха при добавлении в него газа можно не учитывать $Q+G \approx Q$.

Нестационарная задача распространения газа решается при следующих начальных условиях.

- Начальный момент времени $t=0$ - момент увеличения производительности источника тяги, т. е. в момент времени $t=-0$ вентилятор работает в обычном режиме, $Q_{R}^{\prime}=0$ и $Q=Q_{R}$, а в следующий момент времени $t=+0-$ в режиме с повышенной производительностью, $Q_{R}^{\prime} \neq 0$ и $Q_{R}=Q+Q_{R}^{\prime}$. Подача свежего воздуха $Q$ при этом не изменяется.

\section{СПИСОК ЛИТЕРАТУРЫ}

1. Разработка энергосберегающих технологий обеспечения комфортных микроклиматических условий при ведении горных работ / Б.П. Казаков, Л.Ю. Левин, А.В. Шалимов, А.В. Зайцев // Записки Горного института. - 2017. - Т. 223. - С. 116-124.

2. McPherson M.J. Subsurface ventilation and environmental engineering. - London: Chapman \& Hall, 2009. - 824 p.

3. Бокий Б.В., Алабьев В.Р., Тынына С.В. Методика обоснования параметров способа снижения температуры воздуха шахтной атмосферы призабойной зоны тупиковых подготовительных
- Концентрация газа в исходящей струе воздуха в начальный момент времени задана $C_{R}{ }^{(0)}=G / Q$.

Решение задачи производится методом дискретизации, при котором время $t$ разбивается на интервалы, за которые воздух совершает целое количество оборотов по замкнутому рециркуляционному контуру $T$, c: $t_{0}=0, t_{1}=T, t_{2}=2 T, \ldots, t_{n}=n T$. В момент времени $t_{0}=0-C_{R}{ }^{(0)}=G / Q$, при $t_{1}=T$, концентрация газа будет складываться из двух составляющих: 1) смешивания двух потоков - чистого и загазованного воздуха и 2) газовыделения в поток воздуха с увеличенным расходом:

$$
C_{R}^{(1)}=\frac{Q \cdot 0+Q_{R}^{\prime} \cdot C_{R}^{(0)}}{Q+Q_{R}^{\prime}}+\frac{G}{Q+Q_{R}^{\prime}}=\frac{Q_{R}^{\prime} \cdot C_{R}^{(0)}}{Q+Q_{R}^{\prime}}+\frac{Q \cdot C_{R}^{(0)}}{Q+Q_{R}^{\prime}}=C_{R}^{(0)},
$$

что и требовалось доказать - концентрация газа не изменилась. На всех последующих интервалах времени будет то же самое - концентрация газа в тупиковой выработке при увеличении производительности вентилятора с подсосом исходящей струи не меняется.

\section{Заключение}

На основании результатов проведённого анализа можно сделать вывод, что теплоотводящий потенциал породного массива не высок и приводит к быстрому увеличению температуры воздуха в тупиковой выработке с мощным источником тепловыделения. Величина расхода воздуха для выноса вредных примесей может быть недостаточна для нормализации теплового режима. В этом случае целесообразно использование вентилятора местного проветривания с производительностью, превышающей подачу свежего возду$\mathrm{xa,} \mathrm{которое} \mathrm{не} \mathrm{приводит} \mathrm{к} \mathrm{увеличению} \mathrm{загазованности}$ тупиковой выработки, несмотря на то, что часть исходящей струи направляется обратно. Концентрация газа остаётся такой же, как в случае работы вентилятора с производительностью, равной расходу свежего воздуха, проходящего по сквозной горной выработке. При этом интенсивность выноса теплоты даже с учётом частично-повторного использования воздуха возрастает в результате теплообмена со встречным воздушным потоком.

Представленные в статье результаты моделирования тепловой и газовой обстановки в тупиковой выработке могут быть использованы для прогнозирования скорости возрастания температуры воздуха в забое при работе комбайна, а также для оценки необходимого количества вентиляционного воздуха для нормализации проветривания выработки по тепловому фактору.

выработок // Вестник Забайкальского государственного университета. - 2014. - № 12 (115). - С. 4-11.

4. Казаков Б.П., Шалимов А.В., Исаевич А.Г. Адвективное проветривание тупиковой выработки // Горный информационноаналитический бюллетень (научно-технический журнал). 2015. - № S7. - C. 76-82.

5. Amano K., Mizuta Y., Hiramatsu Y. An improved method of predicting underground Climate // International Journal of Rock Mechanics and Mining Science \& Geomechanics Abstracts. 1982. - № 19. - P. 31-83. 
6. Вишневская Н.Л., Лискова М.Ю., Плахова Л.В. Проветривание тупиковых горных выработок как способ нормализации условий труда // Актуальные проблемы охраны труда и безопасности производства, добычи и использования калийномагниевых солей: Материалы I Международной научнопрактической конференции / под ред. Г.З. Файнбурга. Пермь: Пермский национальный исследовательский политехнический университет, 2018. - С. 344-350.

7. Analysis of regularities and reasons of accidents / W. Sheng, Z. Sheng, M. Gao, L. Yang // Progress in Mine Safety Science and Engineering II. - USA: CRC Press, 2014. 800 p.

8. Kirsch P., Shi M., Sprott D. Riskgate: industry sharing risk controls across Australian coal operations // Australian Journal of Multi Disciplinary Engineering. - 2014. - V. 11. - Iss. 1. - P. 47-58.

9. McPherson M.J. The analysis and simulation of heat flow into undergrounds airways // International Journal of Mining and Geological Engineering. - 1984. - V. 4. - P. 165-196.

10. Construction of air flow heat transfer coefficient and calculation of airflow temperature in mine wet roadway / $\mathrm{Z}$. Li, T. Wang, M. Zhang, J. Jia, L. Lin // Meitan Xuebao. - 2017. - V. 42. № 12. - P. 3176-3181.

11. Кремнёв О.А. Теплообмен между вентиляционной струёй и горными массивами старых шахт и выработок // Труды Института теплоэнергетики Академии наук УССР. - 1954. № 10. - C. 12-17.

12. Казаков Б.П., Шалимов А.В., Гришин Е.Л. Теплообмен вентиляционного воздуха с крепью воздухоподающего ствола и породным массивом // Физико-технические проблемы разработки полезных ископаемых. - 2011. - № 5. - С. 92-100.

13. Соболев С.Л. Уравнения математической физики. - М.: Наука, $1950 .-424 \mathrm{c}$.

14. Ермолаев А.М., Адамков А.В., Ермолаев А.А. Аэрогазодинамика тупиковой выработки // Вестник Научного центра Вост-
НИИ по промышленной и экологической безопасности. 2018. - № 2. - С. 66-72.

15. Мартынов А.А., Майбенко Н.И., Плотникова Ю.А. Программное обеспечение расчетов температуры воздуха в тупиковых выработках глубоких шахт // Безопасность жизнедеятельности. - 2018. - № 2 (206). - С. 32-42.

16. Mackay L., Bluhm S., Van Rensburg J. Refrigeration and cooling concepts for ultra-deep platinum mining // Platinum in transition «Boom or Bust» - The Southern African Institute of Mining and Metallurgy: the $4^{\text {th }}$ International Platinum Conference. - South Africa, 2010. - P. 35-39.

17. Локальная рециркуляция при работе вентилятора местного проветривания / А.И. Петров, Н.И. Алыменко, А.А. Каменских, А.В. Николаев // Актуальные проблемы повышения эффективности и безопасности эксплуатации горношахтного и нефтепромыслового оборудования. - 2016. - № 1. - С. 202-208.

18. Моряков П.В. Численное моделирование процесса перепуска отработавших газов дизельной энергоустановки в тупиковую выработку // Вестник Московского автомобильно-дорожного государственного технического университета. - 2011. № 3 (26). - C. 37-41.

19. Comprehensive and integrated mine ventilation consultation model / Cheng Jianwei, Wu Yan, Xu Haiming, Liu Jin, Yang Yekang, Deng Huangjun, Wang Yi // Tunneling and Underground Space Technology. - 2015. - V. 45. - P. 166-180.

20. Красноштейн А.Е., Казаков Б.П., Шалимов А.В. Моделирование нестационарных процессов распространения газовых примесей по выработкам рудника в условиях рециркуляционного проветривания // Физико-технические проблемы разработки полезных ископаемых. - 2006. - № 1. - С. 95-101

Поступила: 13.10 .2019 2.

\section{Информация об авторах}

Казаков Б.П., доктор технических наук, главный научный сотрудник отдела аэрологии и теплофизики Горного института УрО РАН филиала Пермского федерального исследовательского центра УрО РАН.

Шалимов A.B., доктор технических наук, ведущий научный сотрудник отдела аэрологии и теплофизики Горного института УрО РАН филиала Пермского федерального исследовательского центра УрО РАН. 
UDC 622.411

\title{
SIMULATION OF THERMAL REGIME DYNAMICS OF DEAD-END MINE WORKINGS WITH HEAT RELEASE SOURCE
}

\author{
Boris P. Kazakov 1 \\ aero_kaz@mail.ru
}

\author{
Andrey V. Shalimov ${ }^{1}$, \\ shalimovav@mail.ru \\ 1 Mining Institute UB RAS, \\ 78-A, Sibirskaya street, Perm, 614007, Russia.
}

The relevance of the research is caused by the need to reduce the air temperature during mining operations in dead-end workings associated with the release of a large amount of heat. Development of ways to normalize the thermodynamic parameters of mine air in the faces based on the construction of predictive mathematical models of change of the thermal regime is a necessary condition for safety in the mining industry.

The aim of the research is to obtain dependences for determining the rate of air temperature increase in a dead-end mine at known values of the intensity of heat release and its removal by a ventilation jet.

Objects: dead-end workings and the surrounding rock mass.

Methods: solution of the problem of non-stationary heat transfer of mine air and rock mass in the conjugate formulation using Laplace transformations; safety assessment on the gas factor of intensification of heat removal from the production by increasing the air supply to the bottom using recirculation.

Results. The authors have analyzed the problem of heat removal, released during the operation of mining equipment in a confined space of mine workings. It is shown that heat absorption by the rock mass and its removal due to ventilation may not be enough to prevent excessive overheating of the air. A mathematical model of the conjugate heat exchange of air and rock is developed, which allows predicting the temperature increase over time depending on the length of production and the total intensity of heat release in it. It is established that heat exchange lasting several hours can be modeled in the approximation of small times, which greatly simplifies the calculated dependences. The calculation of the dynamics of the thermal regime under a set of physical parameters characteristic of mine conditions of the problem, the results of which were the basis of the approximation made and confirmed the danger of a rapid increase in air temperature with insufficient intensity of heat removal from the workings of the ventilation jet. It is proved that if additional heat removal is necessary, it is possible to use traction sources with a large flow rate and air recirculation, without fear of increasing gas content in the bottom hole.

\section{Key words:}

Heat transfer, dead-end mining, rock mass, Laplace transformations, ventilation, recirculation.

\section{REFERENCES}

1. Kazakov B.P., Levin L.Yu., Shalimov A.V., Zaitsev A.V Development of energy-saving technologies for providing comfortable microclimatic conditions in mining operations. Notes of the Mining Institute, 2017, vol. 223, pp. 116-124. In Rus.

2. McPherson M.J. Subsurface ventilation and environmental engineering. London, Chapman \& Hall, 2009. 824 p.

3. Bokiy B.V., Alabyev V.R., Tynina S.V. The methodology for substantiating the parameters of the method for reducing the air temperature of the mine atmosphere of the bottomhole zone of the deadlock preparatory workings. Bulletin of the Transbaikal State University, 2014, no. 12 (115), pp. 4-11. In Rus.

4. Kazakov B.P., Shalimov A.V., Isaevich A.G. Advective airing of a dead-end mine. Mountain Information and Analytical Bulletin (scientific and technical journal), 2015, no. S7, pp. 76-82. In Rus.

5. Amano K., Mizuta Y., Hiramatsu Y. An improved method of predicting underground Climate. International Journal of Rock Mechanics and Mining Science \& Geomechanics Abstracts, 1982 no. 19 , pp. 31-83

6. Vishnevskaya N.L., Liskova M.Yu., Plakhova L.V. Provetrivanie tupikovykh gornykh vyrabotok kak sposob normalizatsii usloviy truda [Airing of dead-end mine workings as a way to normalize working conditions]. Aktualnye problemy okhrany truda bezopasnosti proizvodstva, dobychi $i$ ispolzovaniya kaliynomagnievykh soley. Materialy I Mezhdunarodnoy nauchnoprakticheskoy konferentsii [Actual problems of labor protection and safety of manufacturing, production and use of potassiummagnesium salts. Materials of the I International Scientific and Practical Conference]. Ed. by G.Z. Feinburg. Perm, Perm National Research Polytechnic University, 2018. pp. 344-350.
7. Sheng W., Sheng Z., Gao M., Yang L. Analysis of regularities and reasons of accidents. Progress in Mine Safety Science and Engineering II. USA, CRC Press, 2014. $800 \mathrm{p}$.

8. Kirsch P., Shi M., Sprott D. Riskgate: industry sharing risk controls across Australian coal operations. Australian Journal of Multi Disciplinary Engineering, 2014, vol. 11, Iss. 1, pp. 47-58

9. McPherson M.J. The analysis and simulation of heat flow into undergrounds airways. International Journal of Mining and Geological Engineering, 1984, vol. 4, pp. 165-196.

10. Li Z., Wang T., Zhang M., Jia J., Lin L. Construction of air flow heat transfer coefficient and calculation of airflow temperature in mine wet roadway. Meitan Xuebao, 2017, vol. 42, no. 12, pp. 3176-3181.

11. Kremnev O.A. Teploobmen mezhdu ventilyatsionnoy struey $\mathrm{i}$ gornymi massivami starykh shakht $\mathrm{i}$ vyrabotok [Heat exchange between a ventilation stream and mountain massifs of old mines and workings]. Trudy Instituta teploenergetiki Akademii nauk USSR, 1954, no. 10, pp. 12-17.

12. Kazakov B.P., Shalimov A.V., Grishin E.L. Heat exchange of ventilation air with the support of the air supply trunk and rock mass. Physical and technical problems of mining, 2011, no. 5, pp. 92-100. In Rus.

13. Sobolev S.L. Uravneniya matematicheskoy fiziki [Equations of mathematical physics]. Moscow, Nauka Publ., 1950. 424 p.

14. Ermolaev A.M., Adamkov A.V., Ermolaev A.A. Aerogasdynamics of dead-end production. Bulletin of the Scientific Center of VostNII on industrial and environmental safety, 2018, no. 2, pp. 66-72. In Rus.

15. Martynov A.A., Maybenko N.I., Plotnikova Yu.A. Software for calculating air temperature in dead-end workings of deep mines. Life Safety, 2018, no. 2 (206), pp. 32-42. In Rus. 
16. Mackay L., Bluhm S., Van Rensburg J. Refrigeration and cooling concepts for ultra-deep platinum mining. The $4^{\text {th }}$ International Platinum Conference. Platinum in transition «Boom or Bust»The Southern African Institute of Mining and Metallurgy. South Africa, 2010. pp. 35-39.

17. Petrov A.I., Alymenko N.I., Kamenskikh A.A., Nikolaev A.V. Local recirculation during operation of the local ventilation fan. Actual problems of increasing the efficiency and safety of mining and oilfield equipment, 2016, no. 1, pp. 202-208. In Rus.

18. Moryakov P.V. Numerical simulation of the process of transferring exhaust gases from a diesel power plant to a dead end.
Bulletin of the Moscow Automobile and Road State Technical University, 2011, no. 3 (26), pp. 37-41. In Rus.

19. Cheng Jianwei, Wu Yan, Xu Haiming, Liu Jin, Yang Yekang, Deng Huangjun, Wang Yi Comprehensive and integrated mine ventilation consultation model. Tunneling and Underground Space Technology, 2015, vol. 45, pp. 166-180.

20. Krasnoshtein A.E., Kazakov B.P., Shalimov A.V. Modeling of unsteady processes of the distribution of gas impurities in the mine workings in the conditions of recirculation ventilation. Physical and technical problems of mining, 2006, no. 1, pp. 95-101. In Rus.

Received: 13 October 2019.

Information about the authors

Boris P. Kazakov, Dr. Sc., chief researcher, Mining Institute UB RAS.

Andrey V. Shalimov, Dr. Sc., leading researcher, Mining Institute UB RAS. 